\section{LA ESCALA GEOGRÁFICA DE LA EXCLUSIÓN EN SANTIAGO DE CHILE. UN ANÁLISIS TERRITORIAL DE LA POLÍTICA PÚBLICA DE REGENERACIÓN BARRIAL Y MEJORAMIENTO DE RESULTADOS ESCOLARES ${ }^{1}$}

Fernando Campos Medina²

\section{THE GEOGRAPHIC SCALE OF EXCLUSION IN SANTIAGO, CHILE, A TERRITORIAL ANALYSIS OF PUBLIC POLICIES ON NEIGHBORHOOD REGENERATION AND IMPROVEMENT OF SCHOOL ACHIEVEMENT ${ }^{1}$}

Fernando Campos Medina²

\begin{abstract}
A growing number of public policies are defining the local scale as the privileged space for State intervention. By observing the spaces of intervention identified by different initiatives at local level, we find that there is rarely territorial commonality between them. What is happening there? In general terms, this has been diagnosed as an example of a lack of coordination in terms of State intervention.
\end{abstract}

This paper holds an alternative explanation arguing that: the lack of spatial congruency is the result of a scale-based issue, in which the 
ha alcanzado tal magnitud, que cualquier selección de espacios locales en su interior, resulta plausible pero al mismo tiempo insuficiente para abordar la verdadera extensión de los problemas sociales.

Mediante la descripción de los espacios de intervención definidos para la regeneración de barrios y el mejoramiento de los niveles de aprendizaje en escuelas, ambos dentro del área metropolitana de Santiago (AMS), se avanzará en una discusión sobre la geografía de exclusión social y de las políticas públicas. Como resultado se destaca que la escala de la exclusión es de macro-zonas, esto es una geografía superior al nivel local, delineando zonas concentradoras, extendidas y espacios intersticiales, como espacialidades recurrentes de problemas sociales en el AMS.

\section{PALABRAS CLAVE: EXCLUSIÓN, ESCALAS GEOGRÁFICAS,POLÍTICASPÚBLICAS,REGENERACIÓN DE BARRIOS, SEGREGACIÓN ESCOLAR.}

Fecha de recepción: 31.10.13

Fecha de aceptación: 29.08.14

1 El presente trabajo se enmarca dentro del proyecto de investigación doctoral del autor desarrollado en el Instituto de Urbanismo Europeo de la Universidad Bauhaus de Weimar, bajo la supervisión del profesor de sociología urbana Dr. Frank Eckardt.

2 Alemania. Sociólogo, Magister en Vivienda y Urbanismo. Candidato a Doctor en Urbanismo Universidad Bauhaus de Weimar. Correo electrónico: fernando.campos@uni-weimar.de geographic extension of socio-spatial exclusion has reached a point where any selection of local spaces is possible but insufficient to address the true scope of social problems.

Through the description of the intervention spaces for neighborhood regeneration and improvement of school achievement within the metropolitan area of Santiago (MAS), this paper aims to make progress towards a debate on the geography of social exclusion and public policies. Findings show that the scale of exclusion is based on macro-zones - a geography that is at a higher level than local geography -, which demarcate concentrating, extended and interstitial areas as recurring scenarios of social issues within the MAS.

KEYWORDS: EXCLUSION, GEOGRAPHIC SCALES, PUBLIC POLICIES, NEIGHBORHOOD REGENERATION, SCHOOL SEGREGATION.

Received: 31.10 .13

Accepted: 29.08.14

1 This paper is part of a doctoral research project carried out in the European Institute of Urban Development at the BauhausUniversity Weimar under supervision of Dr. Frank Eckardt, Professor of Urban Sociology.

2 Germany. Sociologist, MSc in Housing and Urban Development. $\mathrm{PhD}$ Candidate in Urban Development, Bauhaus-University Weimar. Email:fernando.campos@uni-weimar.de 


\section{Introducción ${ }^{3}$}

El interés implícito de este artículo es avanzar en la descripción de la fragmentación socio-espacial en el Área Metropolitana de Santiago (AMS) a partir de la escala barrial. La propuesta de investigación propone relevar los espacios de implementación, es decir, la definición de los territorios de intervención de dos políticas públicas. Por un lado, la regeneración de barrios cuyo objeto es revertir el proceso de acelerada degradación urbano y por el otro, la intervención en escuelas con bajo rendimiento escolar que busca elevar los resultados de aprendizaje. La hipótesis subyacente señala que estas dos formas de intervención,

3 El autor agradece la colaboración del geógrafo Gonzalo Arias para la construcción de los mapas así como en la discusión sobre el análisis espacial que propone este trabajo. Una versión inicial de esta investigación fue presentada en conjunto con Gonzalo al encuentro latinoamericano de geógrafos (EGAL, Costa Rica 2011) "¿La Geografía de la Exclusión Social coincide con la Geografía de la Política Pública en Chile?". son capaces de evidenciar una geografía de la exclusión a través de sus espacios de implementación para el AMS. Esta geografía de la exclusión, desde una perspectiva teórica, debe ser entendida no sólo como una condición estructural del territorio metropolitano, sino también, como la actualización continua de prácticas sociales tendientes a la relegación ${ }^{4}$.

Como señalamos en el párrafo anterior, este artículo observa los espacios de implementación de los programas de intervención para elevar los logros de aprendizaje en escuelas con bajos resultados SIMCE denominados "Escuelas Críticas" (hasta el año 2004) y "Escuelas Prioritarias" (desde el año 2005 hasta

4 “[...] problemas de aislamiento de los pobres urbanos y los mecanismos que nutren y sostienen estas situaciones más allá de lo meramente económico o material." Definiendo aislamiento como "vínculos frágiles - y en último extremo inexistentes - entre los pobres urbanos y las personas e instituciones que orientan su desempeño por las normas y valores dominantes en la sociedad en un determinado momento histórico." (Katzman 2001, p. 172).

5 Sistema de Medición de la Calidad de la Educación. 
2009) por el Ministerio de Educación 6 . Y el programa de intervención en barrios con un alto grado de deterioro físico y social "Quiero Mi Barrio", QMB, (Enero de 2007) desarrollado por el Ministerio de Vivienda y Urbanismo ${ }^{7}$.

6 Los dos programas mencionados hacen referencia al conjunto de programas tendientes a "apoyar de forma focalizada" a los establecimientos que se entienden bajo el concepto de "prioritarios" definidos por MINEDUC. "Aquel establecimiento de educación general básica que atiende a estudiantes de nivel socio económico bajo y medio-bajo, que presenta resultados de aprendizaje deficientes como tendencia persistente en el mediano plazo. Asimismo, mantiene una tasa de repitencia superior a la media nacional y regional, y una sostenida disminución de su matrícula; es común que tenga más vacantes que postulantes y que acepte o le sean asignados estudiantes durante todo el año escolar y tenga una alta rotación de estudiantes" (Mineduc, 2006 citado por Maureira, sf).

$7 \quad$ El programa chileno de regeneración de barrios promovido por el Ministerio de Vivienda y Urbanismo (MINVU) fue denominado "Quiero mi Barrio", siendo también conocido como "200 barrios" en alusión a la cantidad de unidades intervenidas como manera de celebrar el Bicentenario de la Independencia Nacional ocurrida en 1810. El programa surge como una de las medidas para los cien primeros días de Gobierno de Michelle Bachelet en 2006. “El objetivo general del programa es contribuir al mejoramiento de la calidad de vida de los habitantes de barrios que presentan problemas de deterioro urbano y vulnerabilidad social, a través de un proceso participativo de recuperación de los espacios públicos y de los entornos urbanos de las familias. Los objetivos específicos del programa son: recuperar espacios públicos deteriorados, mejorar condiciones del entorno, fortalecer las relaciones sociales y propiciar barrios más integrados" (Información oficial MINVU).
La pregunta que surge de inmediato es por qué combinar dos políticas de origen sectorial ${ }^{8}$ en un análisis de la situación de fragmentación asociada a procesos de exclusión socio-espacial en áreas metropolitanas. La respuesta, a grandes rasgos, plantea que su baja coincidencia espacial es evidencia de la fragmentación territorial y la consecuente ex-

8 Estos dos programas fueron fundamentales en los últimos gobiernos de la "concertación de partidos por la democracia", conglomerado político que gobernó Chile entre 1990 y 2010. Con posterioridad el gobierno de centro derecha modificó dichos programas. Sin embargo, su proximidad temporal y corta duración imposibilita un análisis espacio-temporal como el que aquí se propone para el período 2010-2014. 
clusión socio-urbana asociada a ella. El argumento del artículo propone que la baja correlación espacial que muestran diferentes políticas públicas al definir sus espacios de intervención no señala únicamente una falta de complementariedad sectorial. Por el contrario, dice relación con dos fenómenos que presionan a la política pública desde la dimensión territorial: i) Una imposibilidad institucional de operar de manera integrada en el territorio, lo que significa que la intervención del Estado resulta incapaz para articular "espacios de intervención" y "campos de acción" con el objetivo de lograr acciones de alto impacto; ii) Una problemática social que ha excedido el nivel local o barrial y que por tanto, presenta una complejidad socio-espacial en la forma de grandes áreas o zonas de exclusión, lo que sobrepasa las capacidades de diagnóstico, estratégicas y obviamente, presupuestarias para la acción de la política pública existente.

El presente artículo se organiza en dos secciones, primero una reflexión teórica y posteriormente, un análisis empírico. La reflexión teórica introduce de manera general la reflexión sobre la construcción social de las escalas geográficas. Apoyándose en los que a grandes rasgos se reconoce como la escuela marxista en temas urbanos, la reflexión

$9 \quad$ Espacios de intervención son los lugares donde se despliega un programa de políticas públicas, mientras que campos de acción refiere a las áreas temáticas que pueden ser abordadas por dicho programa. Esta distinción es especialmente clara para el caso de la regeneración de barrios. teórica propone que existiría una línea argumental en el concepto de construcción social del espacio propuesto inicialmente por Lefebvre y la discusión actual sobre construcción social de las escalas geográficas como es destacado por Marston. Como conclusión de esta reflexión se logra liberar un espacio conceptual para el estudio de la exclusión socio-urbana y su propia construcción escalar. En otras palabras, la escala de la exclusión socio-urbana es objeto de estudio, construcción social y no presuposición analítica.

El análisis empírico aborda la descripción de las formas espaciales de la exclusión social a través del análisis de los espacios de implementación de las políticas de regeneración de barrios y de intervención en escuelas con bajos resultados educacionales. Tres dimensiones de análisis son desarrollados: i) coincidencia/no coincidencia espacial de las áreas de intervención, ii) relación espacial entre espacios de exclusión y el nivel socio-económico de sus habitantes, iii) distinción de formas espaciales predominantes para la exclusión socio-urbana con base en la extrapolación de resultados académicos.

Como se ha señalado durante esta introducción, el objetivo general del artículo es describir la 
particular forma espacial que adquiere la exclusión socio-urbana en el AMS. Esto observando cómo los lugares tradicionales de integración social se han vuelto dispositivos de exclusión. Considerando este objetivo, se propone como método un análisis espacial y se descartan otras metodologías como podrían ser las aproximaciones tradicionales de las ciencias sociales basadas en métodos cualitativos o cuantitativos. El supuesto detrás de esta selección metodológica plantea que el estudio de la forma espacial de la exclusión socio-urbana es un primer paso en el análisis de este fenómeno y entrega información indispensable para futuras investigaciones basadas en las técnicas antes señaladas.

Este artículo busca complementariedades y una de ellas es la fructífera investigación que ha desarrollado el Instituto de la Vivienda de la Universidad de Chile en recuperación de barrios tanto a nivel nacional como internacional ${ }^{10}$. Creemos que a partir de dichas experiencias es posible motivar mayores esfuerzos para abordar temas centrales de la política pública territorial a nivel nacional como son: i) un estudio sobre la geografía educacional, ii) la posibilidad en de avanzar en la generación de

10 Campos Medina, García-Ferrando, Larenas y Simó, 2009; Garcia-Ferrando, 2008, Eckardt, 2011. políticas públicas nacionales que usen al territorio como elemento articulador, iii) describir, analizar y comprender la multiescalaridad de los problemas socio-espaciales, iv) generar mecanismos diagnósticos multi-nivel y respuestas acordes a las geografías que se dibujan y desdibujan en el territorio.

\section{Construcción social de escalas y coherencia territorial}

La construcción social de escalas geográficas no es una dimensión analítica que sea comúnmente utilizada dentro de la investigación socio-urbana en latinoamericana ${ }^{11}$. No obstante, en esta línea existen esfuerzos de conceptualización desde los años 60 en el trabajo internacional. El concepto de "construcción social del espacio" de Henri Lefebvre ${ }^{12}$ es un ejemplo de ello y se puede considerar como uno de los primeros esfuerzos por superar una visión objetivista sobre el territorio cuando señala: i) (El) espacio natural (físico) está desapareciendo [...] ii) toda sociedad -y por lo tanto, todo modo de

11 Díaz Orueta, Rodriguez, Devalle y Lourés, 2003. Estudiando la situación de Buenos Aires, los autores son bastante ilustrativos en su referencia a la construcción diferencial de las áreas de exclusión en un espacio metropolitano. Al mismo tiempo, que cuestionan el estudio de la exclusión social en áreas en exceso delimitadas.

12 Lefebvre, 1991. 
producción con sus subvariantes-, produce un espacio, su propio espacio ${ }^{13}$.

El autor francés plantea que la producción del espacio puede ser rastreada a través de los diferentes tipos de espacios y las formas en que ellos surgen. Es precisamente en este sentido, que se puede sostener que el espacio no es el lugar donde las relaciones capitalistas se despliegan, sino por el contrario, el espacio es un instrumento para el despliegue del capitalismo. Las dos citas a continuación describen claramente este argumento.

"El proyecto que estoy delineando, de cualquier forma, no busca producir un (o los) discursos sobre el espacio, por el contrario, busca exponer la actual producción del espacio analizando las diferentes formas de espacio y las modalidades de su génesis en una única teoría" ${ }^{14}$

"Mi hipótesis es la siguiente: Es el espacio y por el espacio donde se produce la reproducción de las relaciones de producción capitalista. El espacio deviene cada vez más un espacio instrumental". ${ }^{15}$

En el trabajo de Lefebvre no se encuentra una referencia directa a la noción de escalas territoriales, por lo menos, no en las referencias aquí expuestas.

13 Ibíd., p 30-31.

14 Ibíd., p. 16 (traducción del autor).

15 Lefebvre, 1974, p. 223.
Su contribución es más bien indirecta, ya que si aceptamos la idea de que el espacio es construido socialmente, comprenderemos que para lo concerniente a las escalas territoriales, no ocurrirá algo diferente. Abordando de manera directa la construcción social de las escalas, encontramos el trabajo de Marston ${ }^{16}$. Esta propuesta teórica dio lugar a una fuerte discusión en la revista "Progress in Human Geography" durante el año 2001, a partir de la crítica a dicho texto presentada por Niel Brenner $^{17}$ y la contra respuesta de Marston ${ }^{18}$. Más allá del detalle de esta discusión es posible observar cómo hasta el día de hoy distintos autores vuelven al componente "social" tanto de la construcción del espacio como de la construcción de las escalas geográficas ${ }^{19}$.

La propuesta teórica que este artículo abraza se encuentra en un lugar diferente, no trata de una construcción generalizada del espacio por la tecnocracia o el mercado, como sería el caso de Lefebvre. Al mismo tiempo, nuestra propuesta no llega a centrarse de manera exhaustiva al nivel de la "reproducción y el consumo" como sería la aproximación de Marston, cuando señala "En este artículo

16 Marston, 2000.

17 Brenner, 2001.

18 Marston, 2001.

19 En esta línea se puede observar el proyecto teórico del geógrafo humano Benno Werlen, especialmente sus libros "Society action and space : an alternative human geography" (1993) y "Sozialgeographie alltäglicher Regionalisierungen 2" (1997)

revista invi Nº 82 / Noviembre 2014 / Volumen Nº 29: 65-89 
quiero profundizar el argumento de que la escala es constituida y reconstituida alrededor de las relaciones capitalistas de producción, la reproducción social y el consumo, y al mismo tiempo, que entregar atención a cada uno de estos tres grupos de relaciones es fundamental para realmente entender la construcción social de las escalas"20. A pesar de ello, no podemos negar que de muchas maneras nos gustaría aproximarnos a la pregunta por el rol de la "cotidianeidad" en la construcción social de las escalas geográficas.

De la misma manera mantenemos cierta distancia de una lógica centrada primordialmente en lo político-institucional, como sería el caso de Neil Brenner al observar la rearticulación escalar bajo lo que se ha denominado "política de la escala", en relación a la "producción, reconfiguración u oposición a algunos de los aspectos de la organización socioespacial en un, relativamente, limitado espacio geográfco- usualmente etiquetadas como lo local, lo urbano, lo regional, lo nacional y así sucesivamente. En este aspecto singular de la "política de las escalas", la palabra "de" connota una relativamente diferenciada y auto-limitada unidad geográfica"21. Si bien reconocemos la centralidad de la acción del Estado en la construcción escalar, para el caso aquí analizado, la forma espacial de la segregación social aparece como el resultado de un dialogo entre la política

20 Marston 2000, p. 221

21 Brenner 2001, p. 599 (traducción del autor).

72 revista invi № 82 / Noviembre 2014 / Volumen № 29: $65-89$ pública, las tendencias socio-urbanas y la acción individual.

Al abordar la construcción social de las escalas geográficas desde la acción de las políticas públicas, ponemos de manifiesto el conflicto que existe entre la territorialidad con que la acción del Estado comprende la exclusión social y lo que suponemos es la territorialidad estructural de la exclusión socio-urbana. El punto intermedio entre ambas realidades es lo que podemos llamar la forma "experiencial" con que las personas viven la exclusión. Este punto intermedio entre la representación geográfica que hacen las políticas públicas de los problemas sociales y su forma estructural distinguible en el AMS, es lo que el análisis empírico mostrará en la próxima sección.

Por lo pronto podemos señalar que el artículo releva un problema muchas veces no tematizado en la discusión sobre políticas públicas y que denominaremos "la presunción de coherencia temporal y territorial de los problemas sociales". Esto significa que a pesar de la claridad que presentan los procesos sociales de exclusión, por ejemplo, en el espacio local o barrial. Ellos responden a procesos dinámicos de diferenciación social, dónde su consolidación temporal se mueve, o bien oscila, entre: i) sus raíces en decisiones cotidianas que persiguen 
la diferenciación social, o sea, su anclaje en la dimensión de la acción subjetiva y ii) su condición altamente extendida en macro zonas urbanas que es el mejor indicador de su condición estructural, o sea, independiente de las decisiones individuales de personas o familias ${ }^{22}$.

A modo de síntesis, propondremos que una manera de acceder a esta doble dimensión de estructura y agencia ${ }^{23}$ en los procesos de exclusión urbana, resulta evidente al retratar los diferentes horizontes temporales y espaciales con que distintos actores operan al interior de las geografías de la exclusión. Esta suerte de inestabilidad puede ser retratada analíticamente bajo la noción de transitoriedad espacio-temporal de las políticas públicas, concepto que esperamos profundizar en trabajos futuros.

22 Esta doble dimensión de la exclusión urbana en tanto forma estructural del territorio y las instituciones, pero al mismo tiempo como prácticas y estrategias individuales, puede ser abordado, entre otras posibilidades, bajo el concepto de habitus elaborado por Bourdieu: "sistema de disposiciones duraderas y transferibles, estructuras estructuradas predispuestas a funcionar como estructuras estructurantes, es decir, como principios generadores y organizadores de prácticas y representaciones que pueden estar objetivamente adaptadas a su fin sin suponer el propósito consciente de ciertos fines ni el dominio expreso de las operaciones necesarias para alcanzarlos, objetivamente "reguladas" $y$ "regulares" sin ser el producto de la obediencia a reglas, $y$, por todo ello, colectivamente orquestadas sin ser el producto de la acción organizadora de un director de orquesta". (Bourdieu, 2007, p. 86).

23 El concepto de estructura y agencia es un elemento clave en la discusión sociológica. Para una visión comprensiva sobre esta tensión en sociología, especialmente desde la escuela inglesa ver Giddens, 1984 y Archer, 1995

\section{Correlaciones espaciales, estructura y agencia}

No es la intención de este artículo concluir de manera simplista que la falta de correlación espacial de los dos programas analizados es un problema de coordinación del Estado. Por el contrario, queremos entender esta diferencia como un indicador de la compleja situación socio-territorial del AMS donde dos sistemas diagnóstico altamente representativos de sus problemáticas no coinciden, por una suerte de desincronización tanto de los "marcos cognitivos de representación”, así como de los 
"horizontes normativos de acción"24 de las políticas públicas. Lo que significa, si se nos permite hipotetizar, que en nuestros "policy-makers" 25 , tanto al nivel cognitivo como normativo, han aceptado la desastrosa transformación que ha significado que los espacios tradicionales de socialización se hayan convertido paulatinamente en dispositivos de exclusión ${ }^{26}$.

Es esta transformación de lo social, lo que ha permitido el surgimiento -entendido como sinónimo de construcción- de una escala en exceso ampliada de la exclusión en AMS. La construcción de esta escala geográfica ampliada de la relegación, no muestra relación únicamente con las capacidades monetarias familiares. Por el contrario, si nos concentramos en el acceso a bienes y servicios por parte de las familias, ha existido indudablemente

24 Ambos conceptos son extrapolados del trabajo de Müller (2000) El autor, en líneas generales, propone que las políticas públicas no son nunca un vehículo para la solución de problemas sociales. Por el contrario, ellas tienen la capacidad de construir marcos conceptuales para interpretar el mundo, los cuales no son unicamente cognitivos sino que movilizan elementos valorativos para la acción.

25 La noción anglosajona de "policy makers" describe a quienes diseñan, desarrollan e implementan las políticas públicas. Comúnmente, el concepto se traduce al español como "hacedores de políticas". El lector debe considerar que bajo la noción de "policy makers" usada en este artículo, la referencia es a todo el proceso de la política pública y no está restringido a ninguna etapa.

26 El argumento que aquí se presenta se basa en una interpretación del enfoque cognitivo en políticas públicas desarrollado por de la escuela francesa. Para una visión comprensiva de esta perspectiva ver Faure, Pollet y Warin, 1995 y Müller, 2000. un mejoramiento y una ampliación durante los últimos años. A pesar de ello, resulta necesario entender que existen grandes áreas o bien macro zonas, en las que opera de manera constante una "vivencia de exclusión" 27.

Nuestro presupuesto implícito en la investigación propondrá que existen familias que viven de manera estable, continua, duradera y predecible en espacios globales que ofrecen bajas posibilidades de integración y altas de relegación. Al mismo tiempo, dichos espacios poseen una escala que no se corresponde a la espacialización de los ingresos o del consumo -como señaláramos antes-, ni tampoco, de las divisiones territoriales definidas administrativamente por el Estado. Por el contrario, la escala de la exclusión tiene que ver con la complementariedad que se produce -estructural así

27 En este sentido se puede entender la intención de Jirón cuando propone complementar el análisis de la segregación en el AMS con las formas invisibles de inequidad. Serían estas formas invisibles las que en este articulo se entienden como la dimensión experiencial de la exclusión, la que sin lugar a dudas no puede ser reducida a formas materiales (Jirón, 2007). 


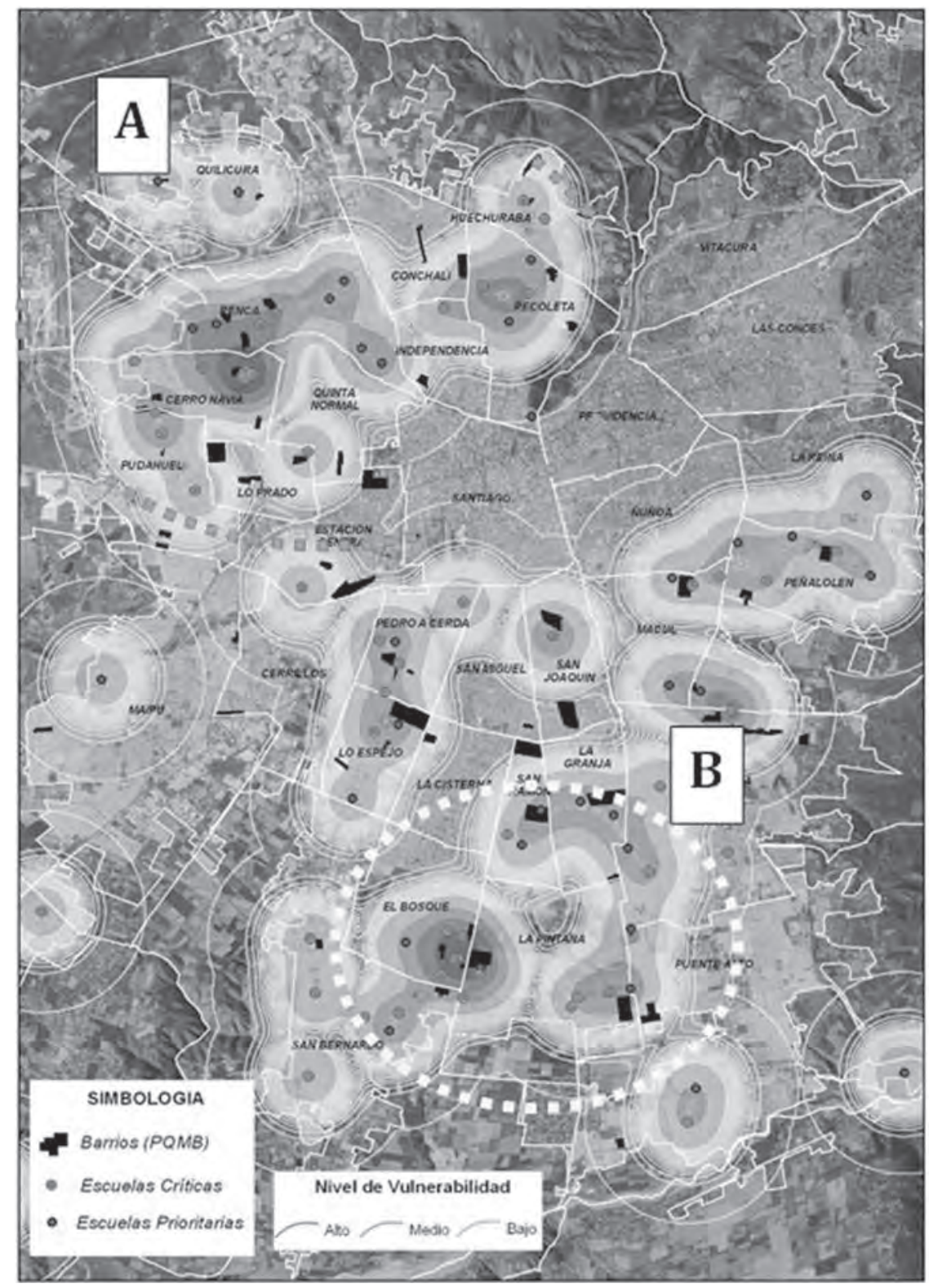

MAPA 1: LOCALIZACIÓN DE ESCUELAS CRÍTICASPRIORITARIAS Y BARRIOS SELECCIONADOS POR EL PROGRAMA OMB

Fuente: Elaboración propia sobre antecedentes MINEDUC 2005 y MINVU 2008. 
como vivencialmente- entre los diversos espacios de socialización que debiendo posibilitar la integración social, por más de treinta años han distribuido desventajas difíciles de superar para numerosas personas, familias y colectivos. Situación que se ha mantenido estable a lo largo de sus vidas.

\section{Análisis empírico}

\section{DESCRIBIENDO LAS MACRO ZONAS DE EXCLUSIÓN}

El siguiente análisis se basa en la descripción de los barrios así como de las escuelas intervenidas para distinguir formas espaciales significativas que permitan entender la geografía de la exclusión. La pregunta que subyace a este esfuerzo señala: Si no es a la escala barrial en la que coinciden barrios y escuelas, ¿en qué escala ellas se articulan de manera de describir una geografía con sentido para la intervención en política pública?

Al comparar la localización de los barrios sobre las que actúa el programa "Quiero mi barrio" (MINVU) y la localización de las escuelas definidas como críticas-prioritarias (MINEDUC) se observan situaciones que pueden resultar contra-intuitivas en un análisis socio-geográfico (ver mapa 1).
A modo general, se destaca una complementariedad locacional de barrios y escuelas, en tanto estrictamente hablando, en la escala barrial, existe una baja coincidencia de espacios de intervención (correspondencia exacta sólo en las comunas de Cerro Navia, San Ramón y la Pintana). Esta complementariedad global no se presenta de manera homogénea en toda la ciudad, si cambiamos la escala y analizamos macrozonas es posible observar que existe una mayor concentración de barrios-escuelas en el norponiente de la ciudad (zona A: Cerro Navia, Renca y Quilicura), que lo observado en el sur-poniente (zona B: La Granja, San Ramón, el Bosque y la Pintana), donde existe mayor cantidad de escuelas en proceso de mejora que barrios en dicha situación. Como observación general, es posible señalar que los barrios tienen un patrón de localización peri-central en la ciudad, mientras las escuelas un patrón mayormente periférico dentro de la trama urbana.

Siguiendo lo planteado por Orellana y Fuentes ${ }^{28}$ encontramos en este primer análisis evidencia para hablar de geometrías espaciales en el AMS que ponen de manifiesto elementos sociales y políticos. i) Es necesario dimensionar que las problemáticas educacionales y el deterioro del hábitat residencial-urbano trascienden los límites administrativos

28 Orellana y Fuentes, 2009 
territoriales en la ciudad ${ }^{29}$. ii) Se muestra con relativa claridad que a nivel de escuelas con problemáticas de aprendizaje, su localización responde débilmente a una determinación comunal y de manera fuerte a geografías urbanas de zonas intersticiales entre-comunas; pudiendo destacarse la frontera sur entre las comunas de El Bosque y La Pintana, el eje Macul-Peñalolén y el clúster Cerro Navia, Renca y Pudahuel. iii) Esta distribución geográfica justifica la necesidad de considerar conceptos como "liderazgo sistémico"30 a la hora de pensar la mejora de resultados de aprendizaje en el AMS. Ello significa hacer frente a la posibilidad de tener grupos de escuelas que muestran lógicas similares, por ejemplo de concentración de alumnos con problemas de aprendizaje, y al mismo tiempo, escuelas que concentrarán a los alumnos aventajados. Todo esto mostrará que mejoras a nivel de escuela y no de grupos de escuelas, puede estar fuertemente influenciado por el efecto localización. iv) Por último, si bien los barrios presentan

29 En relación a la regeneración de barrios, la sustentabilidad de las intervenciones estarán vinculadas a los plazos de intervención, las áreas de acción, pero tambien la institucionalidad existente. La necesidad de superar los límites administrativos comunales estaría en esta lógica. Para un análisis detallado de la sustentabilidad de las intervenciones barriales en el caso chileno ver Sepúlveda y Larenas, 2010

30 Liderazgo sistémico refleja cómo los directivos de un mismo distrito trabajan integradamente para que el mejoramiento de una escuela no se genere a costa de las deterioro de otras del sector, para profundizar en este concepto ver Centro de Estudios de Políticas y Prácticas en Educación (CEPPE), 2009. en alta proporción una localización coincidente con el límite comunal, ellos no aparecen formando una agrupación espacial clara.

Por otro lado, cuando analizamos los barrios y escuelas de manera complementaria, ellos coinciden en la identificación de macro-zonas de interés para intervenciones integrales ${ }^{31}$. Dichas zonas no caen dentro de una clasificación comunal sino que por el contrario describen áreas inter-comunales como son i) la zona nor-poniente respecto a la comuna de Santiago, ii) la macro zona sur y iii) el eje suroriente dividido en el sistema Macul-Peñalolén y Macul-La Florida.

Con relación a los mecanismos de selección de unidades de intervención, el programa QMB utilizó mayormente la opinión de los técnicos comunales, mientras que las escuelas fueron definidas de acuerdo a resultados SIMCE. En otras palabras, podemos decir que las escuelas responden a dinámicas sociales de segmentación y exclusión

31 Ver el análisis de Zapata y Arias (2008) para zonas de interés en materia de renovación urbana usando la relación entre los espacios definidos por el programa Quiero Mi Barrio y los servicios urbanos existentes. 
MAPA 2: ESCUELAS VULNERABLES, BARRIOS Y GRUPOS SOCIOECONÓMICOS: GEOMETRÍAS ESPACIALES, AÑOS 2005 Y 2009
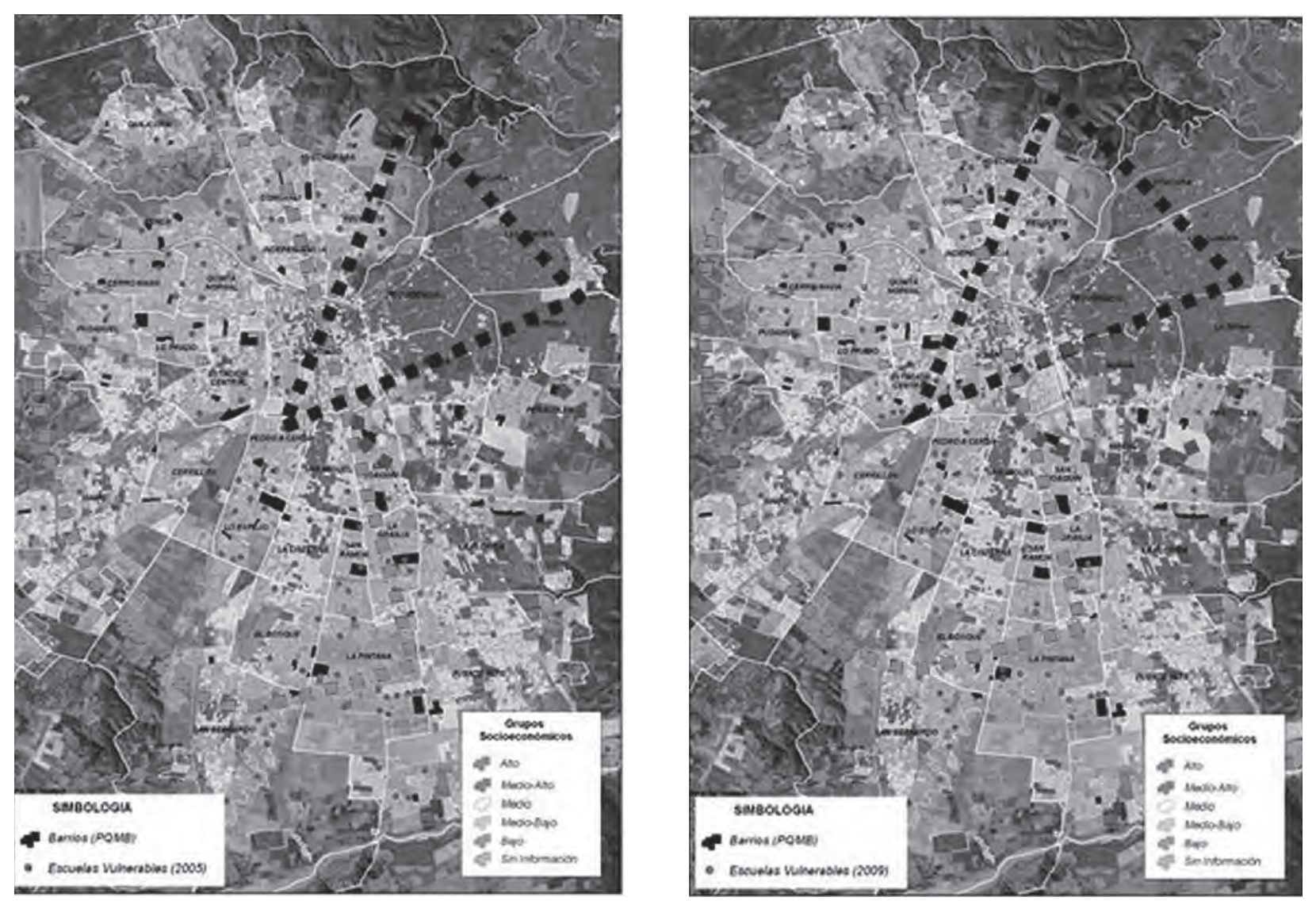

Fuente: Elaboración propia sobre antecedentes MINEDUC, 2005 y 2009. 
educacional, mientras que los barrios responden a desafíos políticos de los municipios sin ser producto necesario de las dinámicas socio-urbanas. Pensar en una vinculación de ambas políticas en una acción con potencial transformador en la ciudad, supone incluir los problemas educacionales como un campo de acción dentro de la regeneración barrial, esto quiere decir observar: i) el nivel educacional de los habitantes en los barrios, ii) las brechas educacionales existentes y el tipo de formación de la población, y iii) los logros de aprendizaje de los estudiantes. No es redundante señalar que es necesario contar con una referencia directa tanto a los domicilios de los estudiantes, como a su rotación entre escuelas para diagnosticar con claridad el condicionamiento urbano de los resultados académicos.

\section{Macro zonas de exclusión y nivel socio-económico}

Los dos mapas que se presentan a continuación analizan los de barrios y escuelas vulnerables para los años 2005 y 2009 respecto de la localización de los grupos socio-económicos predominantes en el AMS.

En términos globales se aprecian relaciones ya clásicas de acuerdo a la idea de territorio-vulnerabilidad. Una baja concentración de escuelas y barrios vulnerables en zonas donde comúnmente se localizan los grupos socioeconómicos altos y una agrupación dispersa en las zonas donde residen los grupos de NSE bajo. El cono invertido de los mapas año 2005 y 2009 (izquierda y derecha respectivamente), muestra que se agregó un pequeño número de escuelas con bajos resultados educacionales en la comuna de Las Condes, la que presenta una favorable situación de conectividad
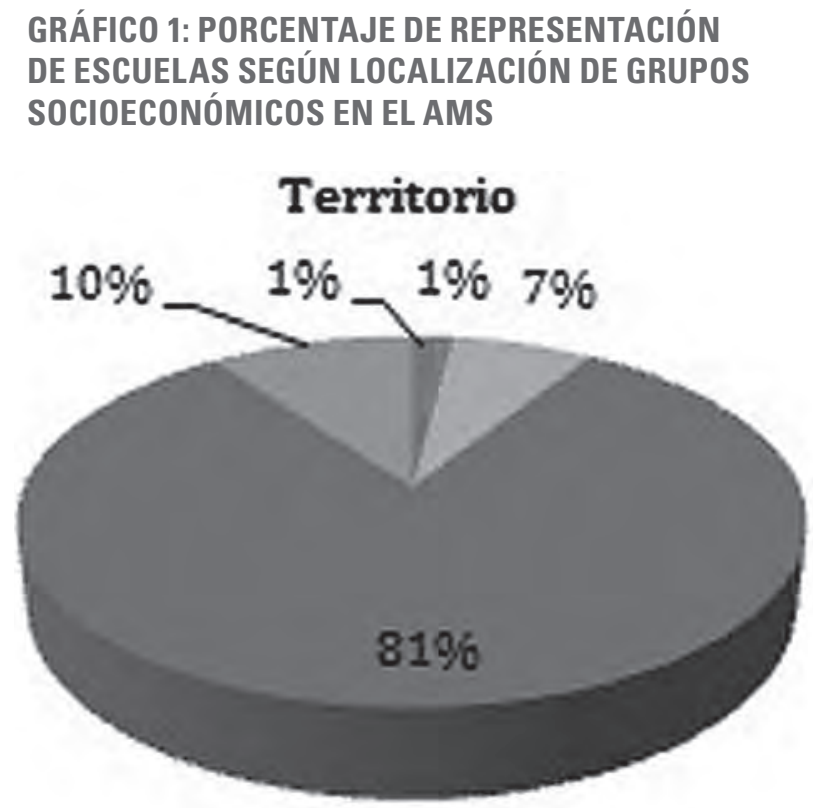

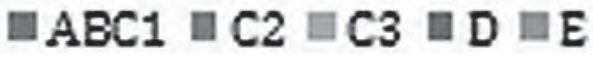

Fuente: Elaboración propia sobre antecedentes MINEDUC 2005. 


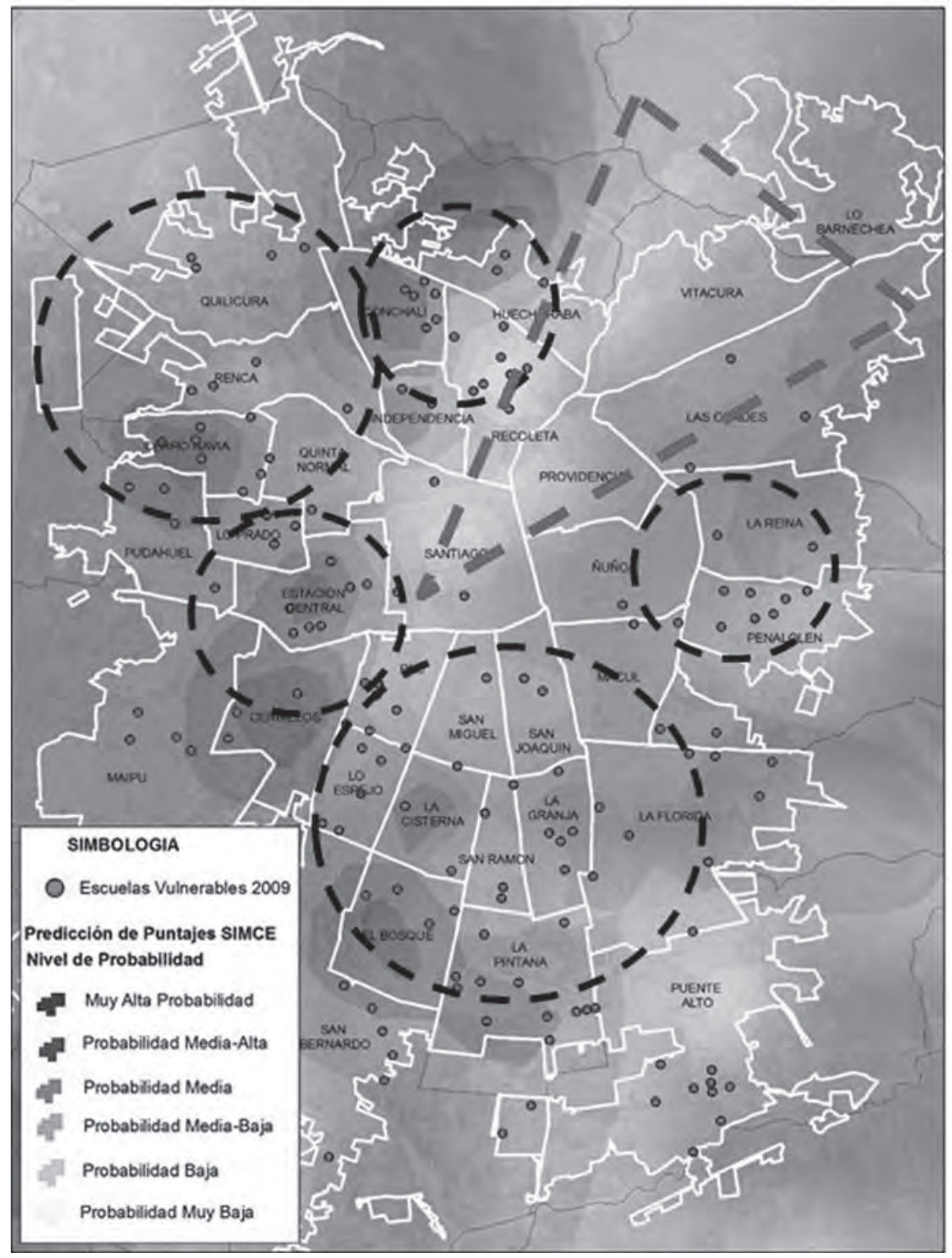

MAPA 3: INTERPOLACIÓN

DE RESULTADOS SIMCE 2009 E IDENTIFICACIÓN DE TERRITORIOS

Fuente: Elaboración propia, sobre an tecedentes MINEDUC - MINVU. 
MAPA 4: INTERPOLACIÓN DE RESULTADOS SIMCE 2009 E IDENTIFICACIÓN DE TERRITORIOS

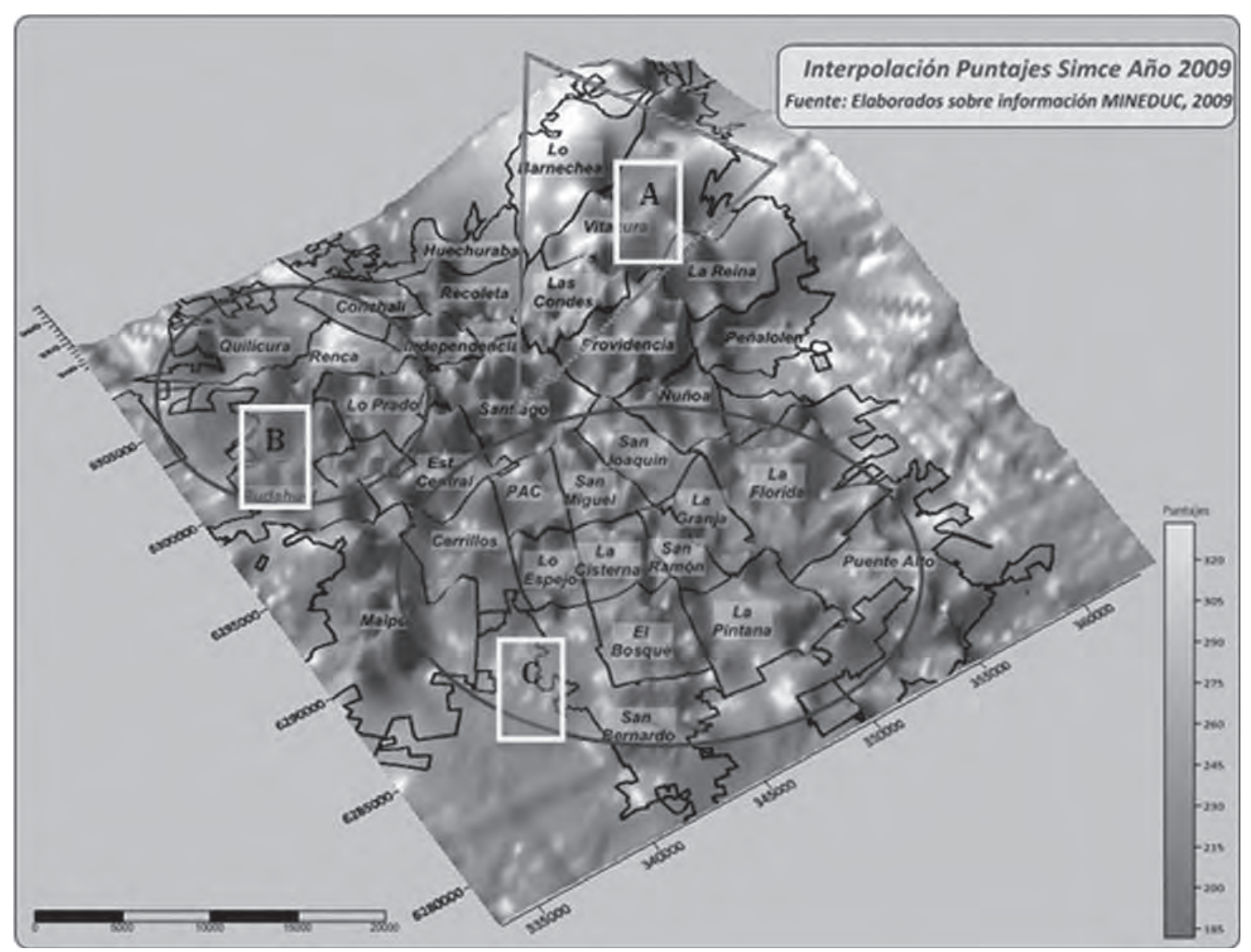

Fuente: Elaboración propia sobre antecedentes MINEDUC 2010.

ARTíCULO: La escala geográfica de la exclusión en Santiago de Chile. Un análisis territorial de la política pública de regeneración barrial y mejoramiento de resultados escolares / 
y accesibilidad urbana, una alta capacidad de gestión y un alto nivel de recursos por habitante. El resto de las geometrías destacadas en los mapas muestran con claridad que las escuelas con bajos resultados académicos se concentran en las áreas ocupadas por comunas con bajo capital, económico y profesional para la intervención.

El gran arco que se extiende desde el sector norte al sector sur-poniente del AMS describe la más alta concentración de escuelas y barrios bajo proceso intervención. En su interior se aprecia el "clúster" de comunas que concentra a los grupos socioeconómicos medio y medios-bajos, macro-zona conformada principalmente desde el norte por las comunas de Conchalí, Renca, Quinta Normal, Lo Prado, Pudahuel, y por el centro-sur las comunas de Pedro Aguirre Cerda, Lo Espejo, San Ramón y La Pintana. El análisis temporal permite observar que son prácticamente las mismas comunas que el año 2005 presentaban la mayor cantidad de colegios es situación de vulnerabilidad, las que vuelven a repetirse el año 2009, con la excepción de Cerrillos y Estación Central que muestran una mejora para el año 2009.

Según se presenta en el siguiente gráfico, el mayor porcentaje de escuelas vulnerables tiende a localizarse en las zonas donde predominan los hogares de los grupos socioeconómicos MedioBajo (D) y Bajo (E), representando en conjunto cerca del $91 \%$ del total. Como se mencionó con anterioridad, existe la presencia de escuelas en zonas donde tradicionalmente se localizan los hogares Alto (ABC1), Medio-Alto (C2), los cuales en conjunto representan sólo el 2\% del universo de establecimientos estudiados. Esta información permite representar una medida diferente de segregación escolar, la cual suma al dato de homogeneidad del grupo socio-económico en las aulas su localización urbana, la cual se muestra igualmente segregada.

\section{Macro zonas de exclusión y resultados académicos}

Con el objetivo de determinar el grado de asociación-disociación de las unidades (escuelas) y la variable en estudio (resultados SIMCE), se procedió a realizar un análisis de interpolación de la variable "puntaje SIMCE año 2009" para el área en estudio. Lo anterior con el fin de distinguir patrones de concentración-dispersión, que a su vez identifiquen macro-zonas 
susceptibles de intervención multisectorial en materia urbano-educacional ${ }^{32}$.

Según el mapa tres, se tiene territorios que se caracterizan por presentar un bajo número de establecimientos educacionales en situación vulnerable $^{33}$, lo que queda representado claramente por el "cono invertido" (A). Situación que se expresa de manera preferente en la macro-zona conformada por las comunas de Santiago, Providencia, Vitacura, Ñuñoa y Las Condes. Es poco probable que en dichos territorios se encuentren valores representativos de la variable, conformando un territorio más bien homogéneo desde el punto de vista de altos logros educacionales. Este resultado pone de manifiesto la relación entre segregación territorial y segregación escolar ${ }^{34}$.

El patrón espacial que caracteriza la presencia de un alto número de escuelas con resultados bajos en la medición del SIMCE 2009, presenta dos dimensiones:

- La zona C evidencia una mayor dispersión territorial de las escuelas con bajos resultados

32 El fenómeno de asociación espacial local es definido por una concentración, en ciertos lugares del espacio analizado, de valores especialmente altos o bajos para una variable en comparación con el valor promedio de ella. La interpolación de los valores de las variables en el espacio geográfico, viene a constituir una herramienta predictiva respecto a un momento dado.

33 Se entiende vulnerables en términos de resultados SIMCE bajo los 220 puntos para el año 2009

34 Atria, 2010. académicos, presentándose como grandes macro-zonas aglutinando sectores particulares de las comunas de La Florida, La Granja, El Bosque, La Pintana, La Florida y Puente Alto, conformando el patrón de resultados académicos del sector Sur de la ciudad.

- Por el contrario, un patrón de concentración en un menor espacio territorial queda definido al analizar en detalle la zona B. Se incluyen aquí sectores de las comunas de Pudahuel, Lo Prado, Renca, Quilicura y Conchalí, caracterizando el sector norponiente de la ciudad.

- La complementariedad de estas dos geografías -descritas anteriormente- es de gran relevancia, ya que muestra la interrelación entre espacios urbanos concentradores de problemas a nivel educacional y otros espacios de efecto extendido. Los espacios concentradores se debieran asumir como producto de dinámicas socio-territoriales distintas a los espacios extendidos. En este sentido, es una tarea pendiente retratar con mayor claridad la geografía 
urbana así como la geografía educacional, pero esta vez geo-referenciando la totalidad de establecimientos educacionales.

- En el entendido que el sistema educacional chileno permite la selección de establecimiento educacional, no es posible señalar que toda escuela acoge sólo a estudiantes de sus alrededores. Por el contrario, resulta más o menos evidente que diferentes establecimientos son mayormente integradores en términos de clases sociales y orígenes urbanos de sus estudiantes que otros. Sin embargo, es poco probable que ello ocurra en los extremos socio-económicos y por tanto, en las comunas y escuelas que claramente acogen a dichos grupos. De este punto se desprende la "paradoja de las escalas" 35 donde se confrontan la escala administrativa del territorio y la escala funcional de los problemas socio-territoriales. En otras palabras, las escala municipal es problemática o bien insuficiente para enfrentar de manera eficiente los problemas educacionales.

\section{Conclusiones}

A través del análisis de los mecanismos de selección de los espacios de intervención para dos políticas públicas, como son los programas de regeneración de barrios y el mejoramiento de los niveles de aprendizaje, se ha querido avanzar en el estudio de las formas geográficas que asumen los procesos de exclusión en el área metropolitana de Santiago de Chile (AMS).

Mediante la identificación de conceptos claves y luego a través de un análisis territorial se ha puesto de manifiesto dos temas de interés: i) Cómo las carencias para la integración social de la población ya no sólo se encuentran a un nivel material, sino que por el contrario, tienen que ver con los espacios de socialización y de construcción del vínculo social y político, como son la escuela y el barrio; ii) Cómo la política pública se ve enfrentada a la complejidad territorial de su intervención, donde ya no sólo es necesaria la focalización y la precisión de cada uno de los mecanismos de diagnóstico territorial, sino que cobran relevancia las capacidades estratégicas de análisis multi-nivel y con ello, la habilidad del aparato Estatal de re-pensar la construcción de geografías socio-espaciales que desafían las definiciones institucionales preexistentes.

En este sentido, la construcción social de las escalas geográficas de la exclusión social aparece como un proceso dinámico. Con bases en los estudios de Lefebvre y Marston, aparece claro que tanto el espacio urbano así como las escalas geográficas son un producto de la sociedad. En este artículo se describe cómo la geografía de la exclusión

35 Sánchez, 2009.

84 revista invi № 82 / Noviembre 2014 / Volumen № 29: 65-89
ARTíCULO: La escala geográfica de la exclusión en Santiago de Chile. Un análisis territorial de la política pública de regeneración barrial y mejoramiento de resultados escolares / 
socio-urbana para el AMS dibuja una forma escalar que no se limita a la escala barrial ni a la escala urbana. Por el contrario, las formas geográficas emergentes de la exclusión aparecen descritas bajo formas extendidas de carácter intercomunal. Ellas alternan en diferentes partes de la ciudad: zonas concentradoras, zonas extendidas y espacios intersticiales como las formas más recurrentes de exclusión socio-urbana.

Bajo esta lectura el análisis de los desafíos de la recuperación de barrios, sientan las bases para pensar en intervenciones socio-territoriales asociadas a la reposición de capitales intangibles como son: el capital social, la participación ciudadana, la movilidad, los logros educacionales, la calificación o re-calificación para el trabajo, etc. Todo ello debiera impactar decisivamente respecto de la reducción de los niveles de segregación socio-espacial. Por otro lado, una estrategia sustentable de mejoramiento en los resultados educacionales pasa por la capacidad de pensar al territorio como un elemento articulador tanto de sus problemáticas como de sus soluciones. Resulta imposible pensar en mejoras continuas de resultados académicos en escuelas aisladas en el espacio barrial, sin analizar las dinámicas

ARTíCULO: La escala geográfica de la exclusión en Santiago de Chile. Un análisis territorial de la política pública de regeneración barrial y mejoramiento de resultados escolares /

Fernando Campos Medina socio-espaciales que ellas conforman y de las que ellas son una consecuencia.

Como observamos en el análisis territorial, la recuperación de barrios en el AMS presenta un patrón espacial diferente pero complementario a las escuelas con bajos resultados educacionales. En una lectura que supera el nivel comunal, se puede apreciar que ambas políticas son sensibles a macro-zonas concentradoras de problemáticas sociales. Ahora bien, en un análisis más detallado de los logros de aprendizaje de las escuelas, se aprecia con mayor claridad que estas macro-zonas se diferencian y redibujan en su interior, destacando: i) zonas concentradoras, ii) zonas expandidas y iii) espacios conectores.

Sin lugar a dudas la capacidad de complementar la intervención en barrios con la intervención en escuelas encierra un fuerte potencial sinérgico ${ }^{36}$. Para ello es imprescindible que cada una de estas políticas utilice información de la otra para la definición de su "espacios de intervención" y "campos de acción”. La regeneración barrial debe incluir resultados educacionales en la definición del barrio a intervenir y por lo tanto, contemplar herramientas para su acción en dicha dimensión. La selección de las escuelas, por otra parte, debe considerar

36 Para observar una buena reflexión sobre lo que se entiende como regeneración barrial sustentable con referencia a los grandes conjuntos de vivienda colectivos en España, ver Pareja y Simó, s.f.

revista invi No 82 / Noviembre 2014 / Volumen Nº 29: 65-89 
elementos de vulnerabilidad relativa a la localización urbana de ellas en, al menos, dos dimensiones: i) las condiciones socio-urbanas del entorno que pueden agudizar los problemas escolares y ii) las dinámicas territoriales de los alumnos propios y de las escuelas cercanas, que pueden impactar en los resultados académicos sólo por efecto de relocalización.

Los resultados aún exploratorios de este estudio son evidencia suficiente para confrontar al menos dos hipótesis ampliamente difundidas en lo referente a la geografía de exclusión en el AMS. Primero, la idea de que segregación en el área metropolitana ha disminuido. Según lo expuesto en este artículo la segregación como experiencia "vivencial" y estructura urbana de los grupos sociales excluidos a nivel residencial y escolar no ha disminuido. La territorialidad de la segregación tendería a mostrar una escala "concentradora" en el área nor-poniente, mientras que reflejaría una escala "extendida" en el sur poniente. Siendo estas zonas "concentradoras", así como las denominados "espacios conectores", los que entregan evidencia a algunos autores que proponer una suerte de disminución de escala de la segregación. Esta disminución escalar, como forma espacial, sería en extremo localizada y no reflejaría la totalidad del fenómeno al nivel metropolitano.

Segundo, los problemas escolares se resuelven en la escuela y en particular en las aulas. Las escuelas de bajos resultados de aprendizaje no son fenómenos sociales aislados espacio-temporalmente, ellos responden a una geografía diferencial de la exclusión respecto de macro zonas en el AMS. Su efecto aglomerado, por localización y relocalización, son elementos que deben ser considerados en intervenciones integrales con la intención de lograr resultados sustentables. En esta línea la idea de crear liceos de "excelencia"37 sería una política equivocada porque justamente desconoce el carácter geográfico de los problemas educacionales. Los establecimientos con problemas de aprendizaje o en condiciones de alta vulnerabilidad social, ven una fuga de buenos estudiantes atraídos por el entorno altamente estimulante de los liceos de excelencia. En términos globales se puede señalar que la sociedad Chilena ha actuado de manera constante a través de políticas públicas altamente focalizadas en la dimensión socio-espacial. Esta situación ha significado una imposibilidad de intervenir en la verdadera geografía de la exclusión social que ha consolidado en las áreas metropolitanas en las últimas cuatro décadas.

37 Liceos Bicentenario, proyecto educacional del gobierno centro derecha que busca generar colegios donde se concentren los mejores estudiates de condición socio-económicas media o baja, ver: http://www.mineduc.cl/index1_int. php?id_portal=57\&id_seccion=3987\&id_contenido $=17572$

ARTÍCULO: La escala geográfica de la exclusión en Santiago de Chile. Un análisis territorial de la política pública de regeneración barrial y mejoramiento de resultados escolares /

Fernando Campos Medina 
Desde una perspectiva holística sería de esperar que la intervención en barrios y escuelas reconociera un potencial de complementariedad tanto al nivel del diagnóstico como de la intervención. Desde este punto de partida, es de esperar que la gran magnitud por el fenómeno de la exclusión social en el AMS, observada desde los espacios tradicionales de inclusión social como son la escuela y el barrio, entregue las pistas a nuestros "policy makers" para definir la correcta territorialidad de la intervención. El presente artículo cuestionó el barrio y comuna como las escalas geográficas adecuadas para iniciar procesos sustentables de mejora. Queda por ver como estos programas evolucionan a partir de 2014 y repiensan la territorialidad apropiada para articular intervenciones que aumenten y mejoren su impacto.

\section{Bibliografía}

ARCHER, Margaret. Realist social theory: the morphogenetic approach. Cambridge, Cambridge University Press. 1995. ISBN-13: 978-0521484428.

ATRIA, Fernando. Mercado y ciudadanía en la educación. Santiago, Flandes indiano. 2010. 125, [1] pp. ISBN: 978-956-8661-00-7

BOURDIEU, Pierre. El sentido de lo práctico. Argentina, Siglo XXI. 2007. 453 pp. ISBN: 978-84-323-1302-8.

ARTíCULO: La escala geográfica de la exclusión en Santiago de Chile. Un análisis territorial de la política pública de regeneración barrial y mejoramiento de resultados escolares /

Fernando Campos Medina
BRENNER, Neil. The limits to scale? Methodological reflections on scalar structuration. [En línea]. Progress in Human Geography. 25(4): 591-614, 2001. ISSN 0309-1325. Disponible en: http://dx.doi. org/10.1191/030913201682688959.

CAMPOS MEDINA, Fernando; GARCÍA-FERRANDO, Lidia; LARENAS, Jorge y SIMÓ, Montserrat. Regeneración urbana en Chile y Cataluña. Análisis de estrategias en fases de diseño e implementación. [En línea]. Cuadernos de Vivienda y Urbanismo. 2(4): 186-209, 2009. ISSN 2145-0226. Disponible en: http://revistas.javeriana.edu.co/index. php/cvyu/article/view/5515.

CENTRO de Estudios de Políticas y Prácticas en Educación (CEPPE). Prácticas de liderazgo directivo y resultados de aprendizaje. Hacia conceptos capaces de guiar la investigación empírica. [En línea]. REICE Revista Iberoamericana sobre Calidad, Eficacia y Cambio en Educación. 7(3): 19-33, 2009. [Fecha de consulta: 28 junio 2010] Disponible en: http://www.rinace.net/reice/numeros/arts/vol7num3/art2.pdf.

DÍAZ ORUETA, Fernando; RODRIGUEZ, Carla; DEVALLE, Verónica y LOURÉS, María Luisa. Ciudad, territorio y exclusión social. Las políticas de recualificación urbana en la ciudad de Buenos Aires. [En línea]. REIS Revista Española de Investigaciones Sociológicas. (103): 159-185, 2003. [Consulta: 27 Junio 2010]. Disponible en: http://www. reis.cis.es/REIS/jsp/REIS.jsp?opcion=articulo\&k titulo $=1900$ \&autor $=F E R N A N D O+D \% C D A Z+O R$ UETA\%2C+CARLA+RODR\%CDGUEZ\%2C+VER 


\section{\%D3NICA+DEVALLE\%2C+MAR\%CDA+LUISA+ LOUR\%C9S.}

ECKARDT, Frank. ¿Cuerpos extraños en el management urbano? La conexión institucional y temática del programa "Soziale Stadt". [En línea]. Revista INVI. 26(72): 187-205, agosto 2011. ISSN 07188358. Disponible en: http://dx.doi.org/10.4067/ S0718-83582011000200007.

FAURE, Alain; POLLET, Gilles y WARIN, Philippe. La construction du sens dans les politiques publiques débats autour de la notion de référentiel. $\mathrm{Pa}$ ris, L'Harmattan. 1995. ISBN: 978-2738435088.

GARCÍA-FERRAND0, Lidia. Retos para un nuevo modelo de intervención en barrios: La Llei de Barris de Cataluña (2004). Cambios en las políticas de regeneración urbana. [En línea]. Scripta Nova. XII 270(58), 2008. ISSN 1138-9788. [Consulta: 28 junio 2010]. Disponible en Internet: http://www. ub.es/geocrit/sn/sn-270/sn-270-58.htm.

GIDDENS, Anthony. The constitution of society: outline of the theory of structuration. Berkeley, University of California Press. 1984. ISBN: 978-0745600079

JIRÓN, Paola. Unravelling invisible inequalities in the city through urban daily mobility. The case of Santiago de Chile. Swiss Journal of Sociology. 33(1): 45-68, 2007. ISSN 0379-3364.

KATZMAN, Rubén. Seducidos y abandonados: el aislamiento social de los pobres urbanos. [En línea]. Revista de CEPAL. (75): 171-189, 2001. ISSN 16820908. [Consulta: 28 junio 2010]. Disponible en: http://www.cepal.org/cgi-bin/getProd.asp?xml=/
revista/noticias/articuloCEPAL/6/19326/P19326. xml\&xsl=/revista/tpl/p39f.xsl\&base=/tpl/topbottom.xslt

LEFEBVRE, Henri. La producción del espacio. [En línea]. Papers: revista de sociología. (3): 219-229, 1974. ISSN 2013-9004. Disponible en: http://papers.uab.cat/article/view/v3-lefebvre

- The production of space. Oxford, UK, Blackwell. 1991. ISBN 0631181776

MAUREIRA T., Fernando. Estrategia de acompañamiento a establecimientos educacionales vulnerables. Cuadernos de Educación. (4), 2008

MARSTON, Sallie. The social construction of scale. [En línea]. Progress in human geography. 24(2): 219-242, 2000. ISSN 0309-1325. Disponible en: http://dx.doi. org/10.1191/030913200674086272

MÜLLER, Pierre. L'analyse cognitive des politiques publiques: vers une sociologie politique de l'action publique. Revue française de science politique. 50(2): 189-208, 2000. ISSN 0035-2950. [Consulta: 28 junio 2010]. Disponible en: http://www.persee.fr/web/revues/home/prescript/article/ rfsp_0035-2950_2000_num_50_2_395464

ORELLANA, Arturo y FUENTES, Luis. El Arco, el cono y los clusters: geometrías espaciales para la gobernabilidad metropolitana y local de Santiago. En: YAÑEZ W., Gloria, ed.; ORELLANA, Arturo, ed.; FIGUEROA, Oscar, ed. y ARENAS, Federico, ed. Ciudad, poder, gobernanza. 
Santiago de Chile, Instituto de Estudios Urbanos y Territoriales, Pontificia Universidad Católica de Chile, 2009. p. 111-132. Serie Geolibros N9; Colección RIDEAL; Eurelibros. ISBN: 978-956-14-0991-0

PAREJA-EASTAWAY, Montserrat y SIMó, Montserrat. What do we understand by a sustainable urban regeneration process? Visions about the future of restructured housing estates in Spain. [En línea]. Restate. s.f. [Consulta: 28 junio 2010]. Disponible en: http://restate.geo.uu.nl/results/visions_spain.pdf

SÁNCHEZ, Joan Eugeni. La gobernanza desde la óptica del poder y las escalas. En: YÁÑEZ W., Gloria, ed.; ORELLANA, Arturo, ed.; FIGUEROA, Oscar, ed. y ARENAS, Federico, ed. Ciudad, poder, gobernanza. Santiago de Chile, Instituto de Estudios Urbanos y Territoriales, Pontificia Universidad Católica de Chile, 2009. p. 21-52. Serie Geolibros N9; Colección RIDEAL; Eurelibros. ISBN: 978-956-14-0991-0

SEPÚLVEDA, Rubén y LARENAS, Jorge. Regeneración urbana. Reflexiones sobre sustentabilidad urbana en el contexto de las estrategias de recuperación barrial en Chile y Cataluña. [En línea]. Cuadernos de Investigación Urbanística. (68): 7082, 2010. [Consulta: 31 octubre 2013]. Disponible en: http://polired.upm.es/index.php/ciur/ article/view/1080

WERLEN, Benno. Society action and space: an alternative human geography. London, Routledge. 1993.
ZAPATA, Isabel y ARIAS, Gonzalo. Impactos urbanos del programa regeneración de barrios, algunas orientaciones claves para la gestión futura. Revista INVI. 23(63): 19-51, 2008. ISSN 07188358. [Consulta: 28 junio 2010]. Disponible en: http://www.revistainvi.uchile.cl/index.php/INVI/ article/view/442/942 